\title{
The Experience of Chemotherapy-Induced Peripheral Neuropathy Among Childhood Cancer Survivors
}

\author{
Caroline Mohrmann, PhD, RN, CPNP-AC ${ }^{\prime}$ (ID
}

\begin{abstract}
As the number of childhood cancer survivors (CCS) is increasing, it is imperative to understand the late effects of childhood cancer therapy to optimize their health and quality of life. Chemotherapy-induced peripheral neuropathy (CIPN) is an unpleasant effect of chemotherapy that affects the peripheral nervous system. This qualitative study uses narrative analysis with a phenomenological influence to understand the lived experience of CIPN among five CCS utilizing photo-elicitation. The lived experience of CIPN is characterized by "a condition of disconnection" with three subthemes: (I) disconnection between mind and body, (2) disconnection between anticipated potential and reality, and (3) disconnection between survivors and support. The condition of disconnection leads to a variety of negative physical and emotional performance outcomes. These findings support the need for refined clinical strategies for identifying this underappreciated condition and further development of interventions to "rebuild the connections" that CCS are lacking.
\end{abstract}

\section{Keywords}

survivorship, symptom management, adolescents and young adults, AYA, late effects of cancer

\section{Introduction}

Childhood cancer survivorship is an emerging field, as 5 -year survival rates have dramatically increased over the past few decades to now over $80 \%$ overall, compared to only $63 \%$ in the 1970 s (Armenian, Landier, Hudson, Robison, \& Bhatia, 2013). With a new, growing, and aging population of survivors, new categories of late complications are being discovered each year. Unfortunately, over $80 \%$ of childhood cancer survivors (CCS) will experience a serious, disabling, or life-threatening health condition by age 45 (Hudson et al., 2013). As a result, there is an increased importance placed on identification of long-term health conditions that result from childhood cancer therapy and continue to affect function or quality of life during survivorship. Acknowledging that knowledge acquisition in this field has greatly improved over the past decades, there are still health conditions experienced by CCS that have not been identified and studied. Chemotherapy-induced peripheral neuropathy (CIPN) is a troublesome symptom experienced by many patients during and after the treatment of cancer, yet we lack knowledge of the lived experience among CCS to support optimal quality of life after the completion of treatment for cancer. CIPN is defined as an injury, inflammation, or degeneration of the peripheral nerves because of the administration of a chemotherapeutic agent (Gilchrist, 2012).

Many patients still experience symptoms of CIPN long after exposure to neurotoxic agents such as vinca alkaloids, platinum-containing compounds, taxanes, and proteasome inhibitors (Armstrong, Almadrones, \& Gilbert, 2005). A recent meta-analysis of over 4,000 adult patients demonstrated that the prevalence of CIPN 1 month after the completion of therapy was $68 \%$, and CIPN often persisted beyond 6 months with a prevalence of $30 \%$ (Seretny et al., 2014). While data are sparse regarding the incidence of CIPN among CCS, it is commonly estimated to be between $25 \%$ and $60 \%$ (Mohrmann, Armer, \& Hayashi, 2016). No agreed-upon diagnostic criteria for CIPN in CCS have been established, which in itself presents a great challenge to the study of CIPN in

'Washington University School of Medicine, St. Louis, MO, USA

Corresponding Author:

Caroline Mohrmann, PhD, RN, CPNP-AC, Pediatric Nurse

Practitioner, Washington University School of Medicine, Department of Pediatrics, 660 S. Euclid Avenue, Campus Box 8I 16, St. Louis, MO 63II0, USA.

Email: mohrmann@wustl.edu 
children and adolescents (Gilchrist, 2012). Evidence in the adult literature supports that CIPN affects vocation, life roles, emotional health, social interactions, sleep, and sexual functioning; even when symptoms are less severe, they can have clinically significant impact on a patient's quality of life (Bakitas, 2007; Driessen, de Kleine-Bolt, Vingerhoets, Mols, \& Vreugdenhil, 2012; Eckhoff, Knoop, Jensen, \& Ewertz, 2015; Ezendam et al., 2014; Tofthagen, 2010a, 2010b; Tofthagen, Overcash, \& Kip, 2012; Wampler et al., 2007). Patient-reported outcomes and clinical significance of functional impacts have not been found to date in the pediatric literature.

When CIPN involves the motor fibers, patients experience symptoms such as weakness, fasciculation, or muscle atrophy (Chung, Prasad, \& Lloyd, 2014). Sensory CIPN causes numbness, tingling, and/or altered perception of pain (Chung et al., 2014). Hence, CIPN can cause both motor and sensory deficits. Functionally, children with CIPN may not be able to open a jar, button their pants, hold a pencil in school, or walk with a normal gait. Those experiencing toxicity during therapy may require dose modifications of the chemotherapeutic agent, increased need for pain medications, and other supportive care interventions. Unfortunately, strong evidence is lacking as to the best options to treat or prevent CIPN in children, likely due to the poor overall understanding of this condition (Gilchrist, 2012; Kottschade et al., 2010). While gabapentin is commonly used in clinical practice, there have been no randomized controlled trials supporting its use (Friedrichsdorf \& Nugent, 2013). This poor understanding includes the trajectory of the CIPN experience during and after therapy, few identified risk factors for developing acute or chronic CIPN, and no identified studies investigating the quality of life or daily lived experience of CCS with this chronic condition. In order to inform the development of diagnostic strategies and improve management of this chronic condition, the following research questions were asked:

Research Question 1: What is the lived experience of painful or function-altering CIPN after the completion of therapy for childhood cancer?

Research Question 2: How does chronic CIPN affect school and/or vocational performance after the completion of therapy for childhood cancer?

Research Question 3: How does chronic CIPN affect the quality of life of CCS?

In order to fully evaluate the scope of the symptom experience, the updated theory of unpleasant symptoms was used as a guide for data collection and analysis (Lenz, Pugh, Milligan, Gift, \& Suppe, 1997). Briefly, the theory of unpleasant symptoms explains the symptom experience in terms of influencing factors that affect a patient's symptom experience, which results in a patient's performance. There are complex, multidirectional relationships between these variables (Myers, 2009). The symptom component of the model comprises distress, timing, intensity, and quality.

\section{Method}

\section{Design}

A narrative study with a phenomenological influence was performed at a large, Midwestern academic medical center. Narrative inquiry within nursing is generally founded on the belief that stories help people share meaning in their life and help clinicians understand how people experience the world (Emden, 1998; Sandelowski, 1991). Phenomenological studies aim to understand the lived experience of a certain phenomenon from the perspective of those living that certain experience, since Husserl proposed that the human experience is valuable and worthy of scientific study (Lopez \& Willis, 2004). While this study was influenced by phenomenology, it was not phenomenological since the data collection strategies were not aimed at the truly "embodied" experience. The goal was to have patients tell their story of their lived experience, which included some detail of how their bodies feel and experience CIPN in their daily lives. This research utilized photo-elicitation, as guided by hermeneutical photography. In hermeneutical photography, "the images captured with photography invite human beings to speak about these experiences with reflective depth. Photographs not only gather interpretations of images of experience, but also enrich and extend the communications of those experiences" (Hagedorn, 1994, p. 46). The purpose of including photographs was to increase our understanding of CIPN by allowing expression of their experience in an alternative format that adds to the spoken word. While the photographs were meant to enrich the data, it was not a required element for participation.

\section{Participants}

All participants were recruited from the Late Effects Clinic for CCS. All the participants met the following inclusion criteria: (1) has completed therapy for cancer at least 1 year prior to study enrollment and is still experiencing CIPN; (2) has a history of childhood cancer diagnosed before the age of 21 years (which is generally the age cutoff to be considered a childhood cancer); (3) is currently between the ages of 14 and 30 years to capture a range of adolescent and young adult survivors typically seen in long-term follow-up clinics; (4) had previous cancer treatment that included neurotoxic chemotherapy, such as vinca alkaloids, platinum-containing compounds, taxanes, and/or proteasome inhibitors; (5) is cognitively 
able to participate in an in-depth interview; and (6) is English speaking. Cognitive ability would be questioned if potential participants did not seem to understand the described study procedures. Those potential participants would be asked to summarize their study participation, in their own words, to assess their understanding. In the event the potential participant could not describe the study procedures, they would be ineligible. Eligible participants were identified by medical record review identifying documentation of CIPN by practitioners in the Late Effects Clinic. Purposive sampling was used and recruitment continued until saturation was reached, which was considered the point at which no new themes or patterns emerged (Sandelowski, 1995). Thus, the ultimate sample size was driven by the data.

\section{Data Collection Procedures}

Institutional review board approval was granted. Recruitment took place within a private exam room in the Late Effects Clinic for survivors of childhood cancer or via phone call. All study procedures were explained, and the participant's questions were answered. All participants provided their verbal informed consent prior to any study procedures. No protected health information or other participant identifiers were collected as a part of this study. A signed consent would be the only document linking a participant identifier to the study; therefore, verbal consent rather than written consent was utilized. After informed consent was provided, participants received a packet that included a copy of the waiver of documentation of consent form, instructions for taking photographs, a 27-exposure disposable camera, and a pre-addressed, prestamped return envelope. Participants were instructed to take photographs that helped to explain their experience of living with CIPN; participants did not need to take all 27 possible photographs. Once the photographic portion was complete, cameras were returned by mail and participants were contacted to schedule an interview. A semistructured interview guide was used for the onetime, in-depth interview, which included review of the participant-generated photographs. The SHOWeD method was used as a model to generate photographic data as a part of the semistructured interview (Catalani \& Minkler, 2010). SHOWeD stands for "(a) What do you See here? (b) What's really Happening here? (c) How does this relate to Our lives? (d) Why does this problem, concern, or strength Exist? (e) What can we DO about it?" (Catalani $\&$ Minkler, 2010, p. 438). During the participant's interview, each photograph was discussed in detail after the completion of the semistructured portion. After the interview, participants were asked for their permission to publish their photographs and informed that their faces would be blurred/pixelated to protect their identity. Each interview was recorded and transcribed verbatim by contracted transcriptionist and reviewed for accuracy by the researcher. During the interviews the researcher was analyzing the participants' expression of their lived experience and then asking focused questions to generate more data (Richards \& Morse, 2012). All photographs were developed in film as well as CD format. Transcripts and photographs were stored in NVivo software for data analysis.

\section{Analysis}

Data analysis was ongoing during data collection. The researcher read each transcript multiple times and reviewed corresponding photographs to immerse herself within the data. The "interpretive engagement" framework was used to analyze participant-generated images (Drew \& Guillemin, 2014). Participants' discussion and interpretation of the images is the first step in analyzing the photographic data. Subsequently, the researcher's reflection and assessment of the images further the analysis. The last step is recontextualizing, considering theoretical frameworks and the audience's perspective. While the participants' first audience to view their photographs is the researcher, additional audiences could be the participants' friends and family, and, eventually, professional audiences. Initial coding of the transcribed interviews began with extracting interpretive statements. The interpretive statements were reorganized and categorized until one major theme and three subthemes emerged; a detailed description of the phenomenon was developed.

\section{Methodological Rigor}

Reflective memoing was performed after each level of coding to further refine the themes and to ensure that the themes were consistent with the researcher's reflection on the representation of the data. To improve trustworthiness, member checking was completed after the final themes had emerged by reviewing and discussing the themes with two participants. No recommendations for modifications were made, but rather, there was confirmation that the developed themes were representative of the research participants' experience. Final themes were shared and discussed with clinicians caring for CCS in a long-term follow-up clinic during a private meeting to allow for expert review.

\section{Findings: A Condition of Disconnection}

Seven participants consented to participate and were assigned a study number (1-7). Two participants did not 


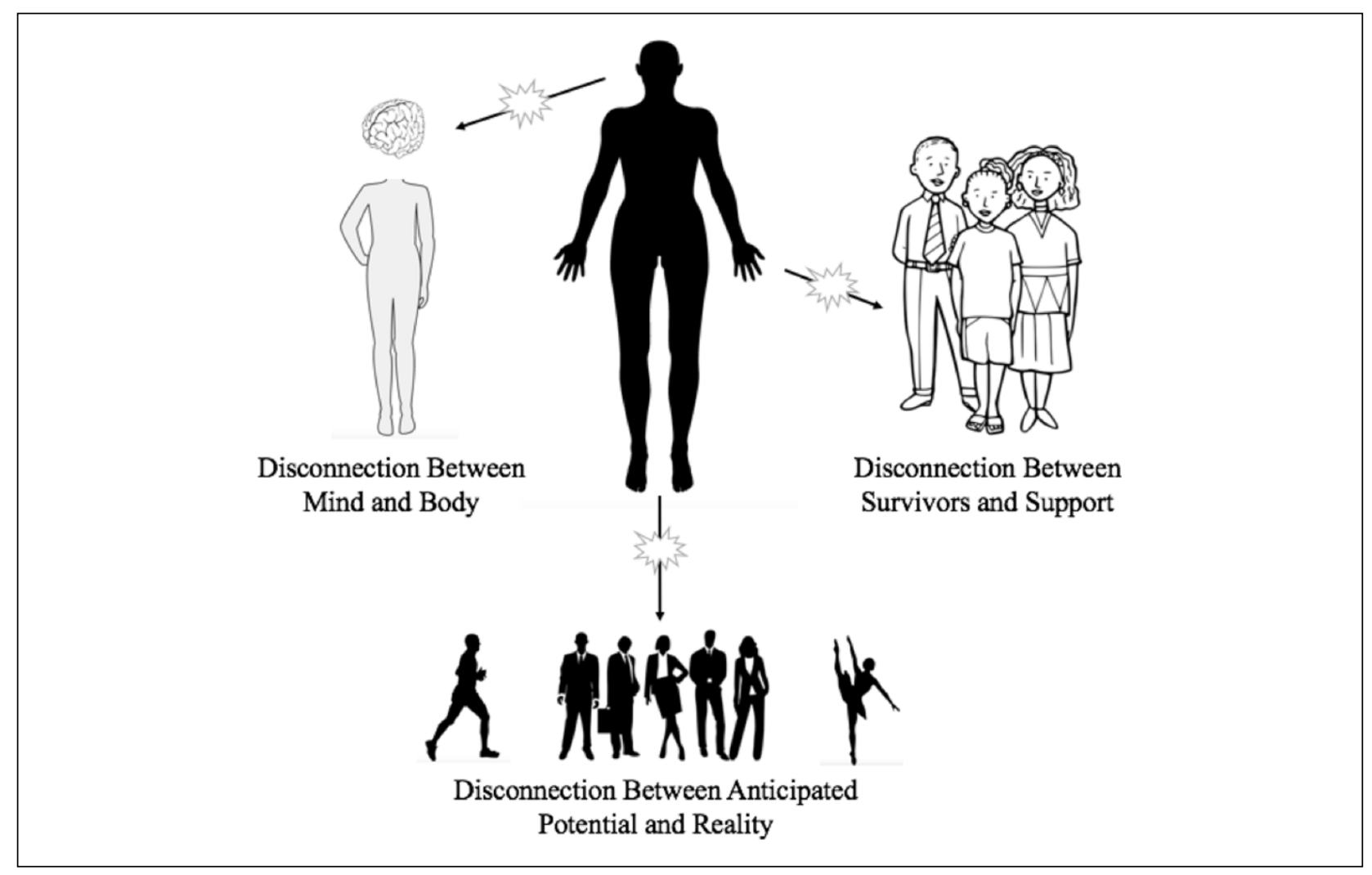

Figure I. The experience of chemotherapy-induced peripheral neuropathy: A condition of disconnection.

complete the study procedures and were not included in the data analysis. A total of five participants ages 18 to 30 years completed both the photographic portion and the interview portion of the study. Four of the participants were female. One participant was African American, and the other participants were Caucasian. The two participants not included in the data analysis were also African American. As a group, the five participants represented a variety of primary cancer diagnoses, three different neurotoxic chemotherapy agent exposures, and radiation therapy, as well as surgical tumor resection. Four participants were employed, and two were students; one participant was a student and an employee. Three participants completed face-to-face interviews, and two participants requested phone interviews. The experience of living with CIPN among CCS is characterized by the overall theme "a condition of disconnection," with three subthemes: (1) disconnection between mind and body, (2) disconnection between anticipated potential and reality, and (3) disconnection between survivors and support (Figure 1).

\section{Disconnection Between Mind and Body}

All participants described CIPN in terms of how their mind often perceived a reality different from what their body was experiencing. Participants described four types of mind-body disconnection: (1) feeling what is not present, (2) not feeling what is present, (3) experiencing pain disproportionate to the amount of aggravation, and (4) not controlling bodily movements as anticipated. While the symptom description in terms of quality, timing, and intensity varied between participants, and even within a participant, each participant's description of the CIPN experience is characterized by a mind-body disconnection. For example, Participant 1 used the words tingling and numbness to describe her symptoms, while Participant 6 used the terms frozen and cramping, which is considered a between-participant variability (Figure 2). Participant 1 clarified that the tingling sensation is felt in her hands, while her feet have numbness, which is considered a within-participant variability.

Feeling What Is Not Present. Participants used a variety of symptom quality terms to describe sensations their mind perceived but that were not actually elicited by what their bodies were experiencing (Figure 3). Some participants reported this experience as feeling "hypersensitive," while all participants reported this as "weird." Below are two participant's descriptions of feeling what is not present: 


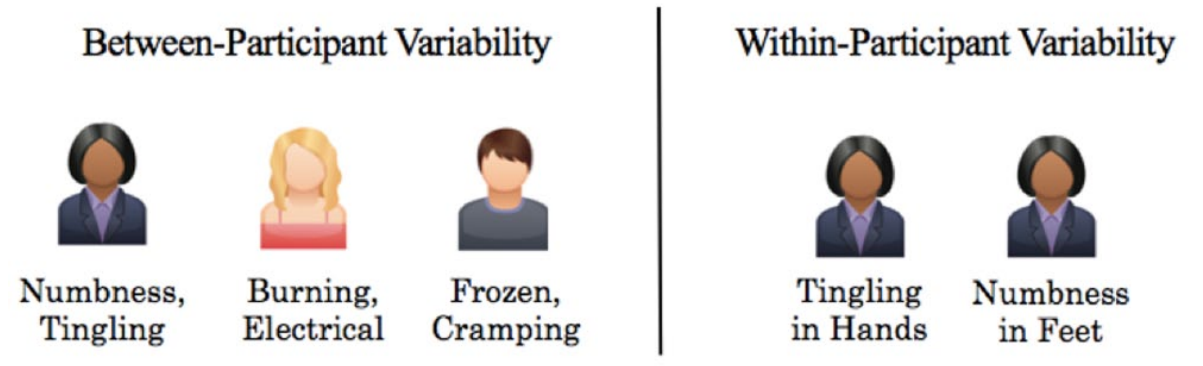

Figure 2. Between- versus within-participant symptom variability.

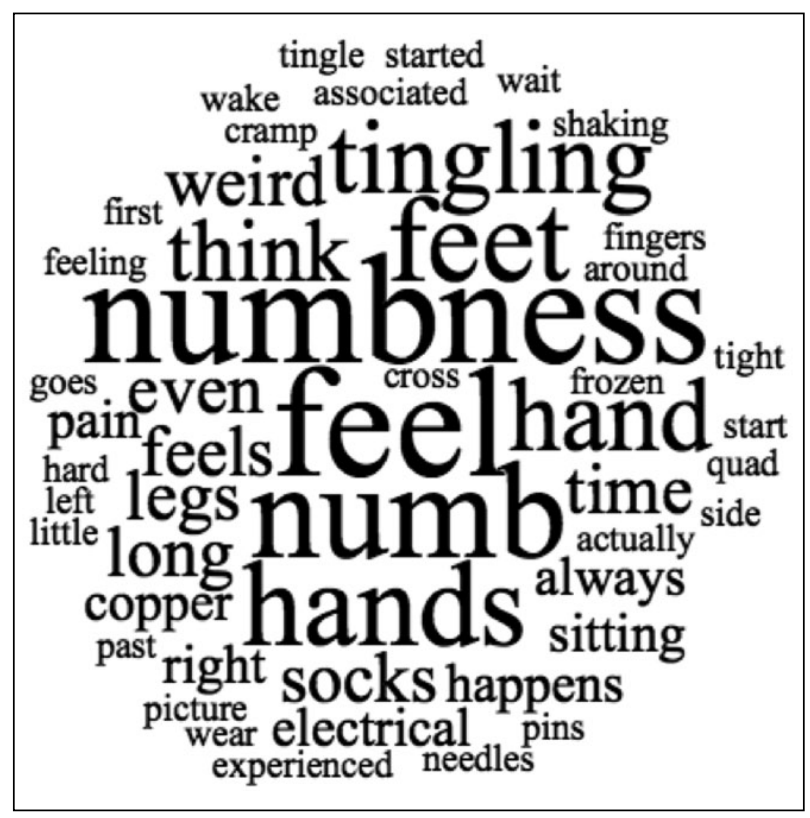

Figure 3. Symptom quality word cloud.

Because that's the main pain that I feel the most intense, for me, is that feeling of just copper, hot copper being pressed into the back of my leg. (Participant 1)

It feels like somebody is pricking me with a pin in my hands. (Participant 5)

Not Feeling What Is Present. Participants also described numbness in a variety of contexts. Sometimes, numbness is a progression from other symptoms of sensory CIPN. Meaning, participants may feel tingling or burning that fades into numbness. Not feeling what is present was most commonly reported in the hands and feet, consistent with the stocking-glove distribution of CIPN. Numbness may be felt after certain activities:

Yeah. So I think whenever it first started it was mostly my hand would just shake, so there was a lot of shaking mostly down in my ring finger and my pinky. And then that kind of

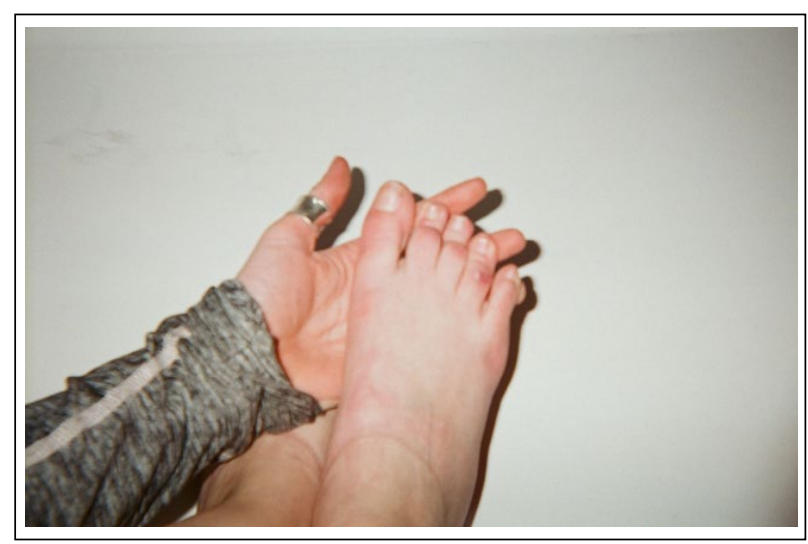

Figure 4. Cellulitis caused by footwear in presence of chemotherapy-induced peripheral neuropathy.

turned more into- - so it still shakes a little bit but it mostly now kind of like a numbness. Sometimes my fingers will go numb and then they'll tingle, especially if I'm doing - like at work. (Participant 4)

A risk associated with lack of sensation is injury. If patients are unable to feel pain, they are unable to appropriately respond to threats to their body. One participant described challenges she faced with injury while working long holiday hours in a retail position. She was on her feet each day and has difficulty finding supportive and appropriate footwear. She was unable to feel her shoe rubbing against her toe over a period of weeks, which led to skin breakdown. Eventually, she required treatment for cellulitis (see Figure 4).

I went to go see my podiatrist the other day and my neuropathy, I almost have, not numbness, but I can feel pain, but I don't feel the symptoms before I get to extreme pain. (Participant 7)

Pain Disproportionate to the Amount of Aggravation. All but one participant reported that they experienced pain. Pain could occur at any time of the day, with or without an identifiable aggravating factor. Common aggravating 
factors were walking, standing, being in any position for prolonged periods, cold or hot temperature, and footwear. Unfortunately, pain could also occur upon waking or when trying to sleep.

Well, I think that that 10-minute walk I had really is what causes me the most pain. But also it's weird because it will happen during the walk, but then I do that at like 2 p.m. I will still be having pain at like 8 p.m. until I go to bed. And it's not like a constant pain, but it will come back and I'll feel it four or five times before I go to bed. So it's kind of continual. It will go away but it comes back. (Participant 1)

And then with my feet, they feel numb or if I wear shoes and socks too long or socks, definitely, socks, if I wear socks too long they make my feet hurt. (Participant 5)

Not Controlling Bodily Movements as Anticipated. As either a result of the sensory symptoms experienced or due to motor CIPN, there were times when participants with CIPN were unable to move and control their bodies as anticipated. As a result, mobility can be limited and rolerelated tasks were difficult to complete.

Yeah, like if I sit down too long, and then I get up, my legs are really sore. And sometimes I kind of look like an old lady whenever I get up, and I just get up and then crouch, because my legs, I guess, kind of asleep, but not asleep, more painful than asleep. (Participant 5)

I know that sounds kind of weird, but since you can't control them, they don't feel like they're there, if that makes sense. It's kind of weird, but more of a lack of awareness and really essentially feeling like you're trying to make something happen and it's not — it almost feels inanimate. (Participant 7)

One participant described feeling disconnected to her body, which sometimes results in feeling off-balance. This led to difficulties in navigating open space. As a coping mechanism, she found that she needed to simply reach out and touch a solid surface to reconnect her mind and body (see Figure 5).

\section{Disconnection Between Anticipated Potential and Reality}

All the participants described ways in which their life had changed due to CIPN. The disconnection between their anticipated potential and reality is seen in their perception of the need to perform their daily functions but their lack of ability to perform those functions as anticipated. Between-participant differences were recognized. Some participants experienced only mild discrepancies between their potential and reality. For example, one participant explained that only a few functions of her daily life could

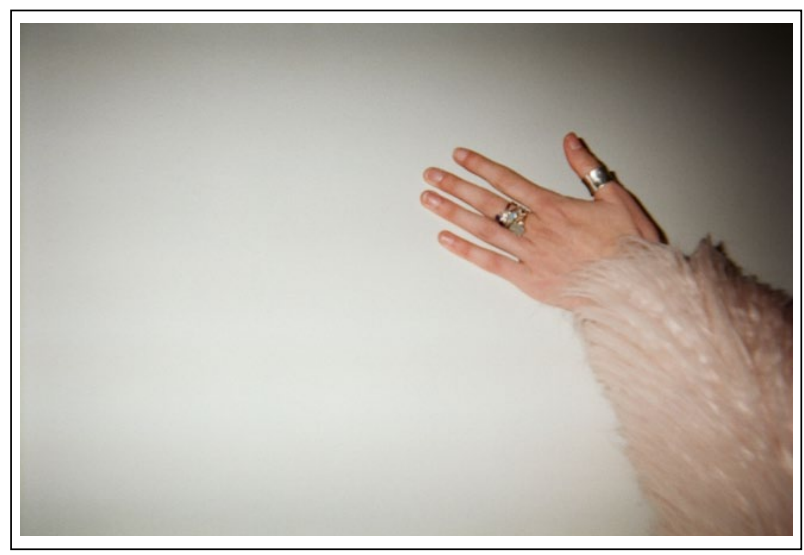

Figure 5. Using touch to reconnect mind and body.

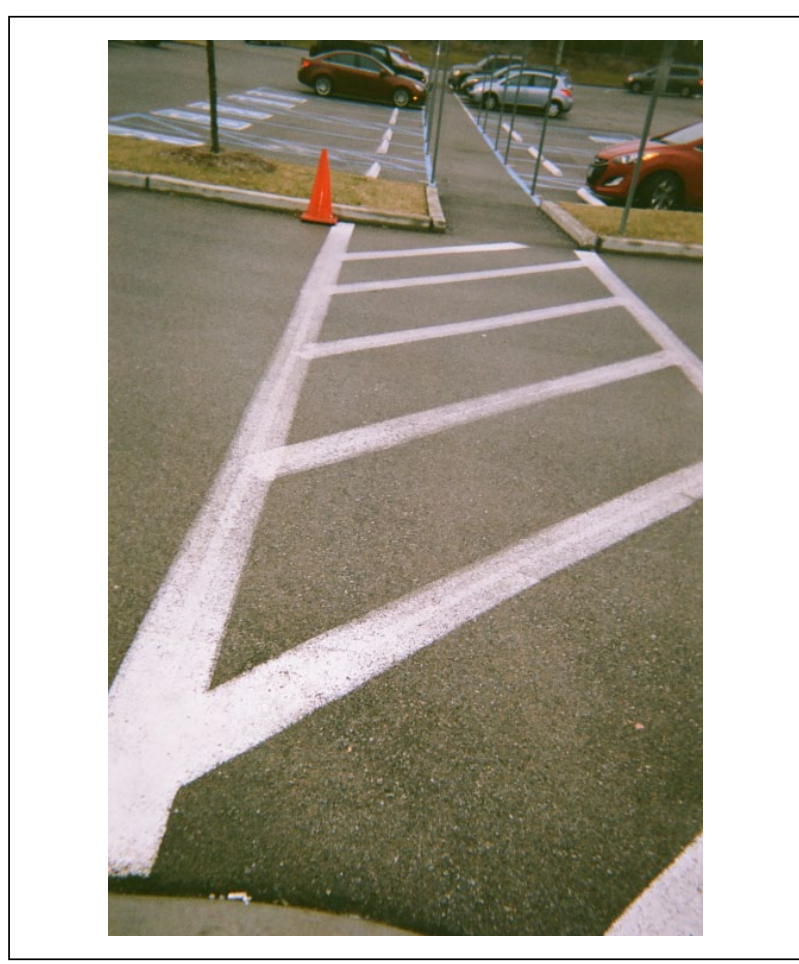

Figure 6. "Am I going to be able to get across today?"

not be performed as anticipated, while another was unsure if she would be able to simply walk a small distance (see Figure 6):

No. It will only get painful when I've been writing or typing for a long period of time, and then it will just cramp up and my hands will be just stuck like this or something. (Participant 3)

Some participants described the symptoms as much more pervasive, to the point where many activities of daily 


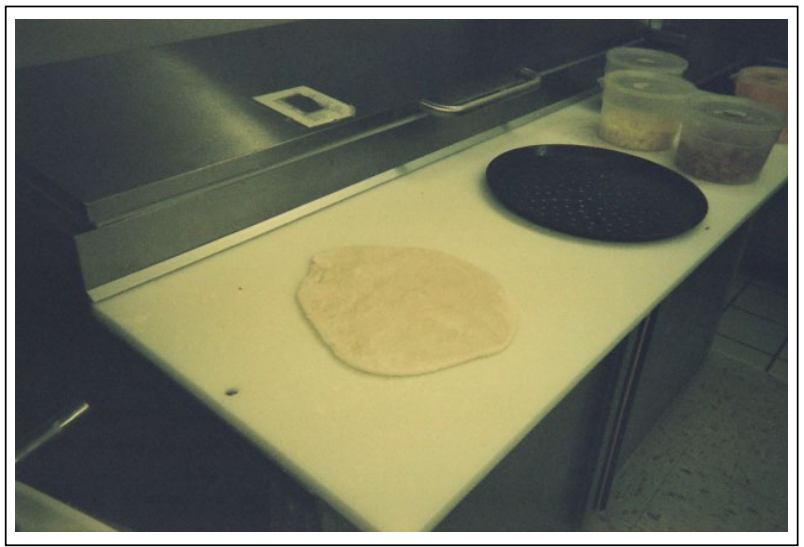

Figure 7. Daunting equipment demonstrating the challenges of work.

living were challenging. At school, participants struggled with walking long distances between classes. At times, they were not able to get to their classroom as anticipated. One participant had to request classroom locations be moved to closer proximity, and another had to resort to only driving around the campus. At home, participants were often unable to complete chores, such as washing dishes or folding laundry. One participant explained that the unexpected timing of the symptoms limited when she was able to take care of her home:

I guess, on a daily basis you have to do different things around the house, and if you're not feeling well, there are some things that you can't do. And then you feel just kind of worthless, I guess. (Participant 5)

The workplace environment led to participant's increased sensory and motor symptoms as well as pain. A common experience in many workplaces, prolonged standing, often elicits pain. The use of equipment can be challenging (see Figure 7). Some equipment requires a level of manual dexterity, and participants who felt disconnection in their hands were often unable to control the equipment to complete work-related tasks. Using equipment that functions with repetitive motions or that is heavy can lead to sensory symptoms and/or pain. Temperature variability at work also caused pain. One participant described having to work in a freezer as a part of daily responsibilities in the food industry, even though coldness led to extreme pain. As a result, CCS living with CIPN are at risk for underemployment or limited career trajectory. One participant described turning down a promotion because it would require her to live in a geographic region with frequent snow, and she is unable to easily walk in winter conditions.

Recreation, romantic relationships, and spiritual expression were also aspects of life where participants often cannot perform as expected. Almost all participants gave examples of leisure activities they did before their CIPN that have now changed. For example, one participant was limited in how long he could play the drums before his hands hurt; one participant loved participating on the bowling team but could now only play three games before her hands hurt; and one participant loved lacrosse but could not run without pain so now she takes yoga classes. Romantic relationships can also be affected, as physical intimacy can be painful as well. One participant reports even holding hands caused pain. Religious practice is affected for those who use their bodies as a part of their spiritual expression but now have difficulty manipulating their bodily movements to maintain a position without pain. One participant reflects that the experience of CIPN has been globally life-altering:

It's kind of like that weird-I don't know where I would be. I think I'd be in somewhere completely different if I didn't have neuropathy or the tumors or anything like that. I think it would be a completely different life path. (Participant 7)

\section{Disconnection Between Survivors and Support}

All survivors voiced frustration that CIPN is difficult to explain and therefore difficult for others in their life to understand. This lack of understanding and appreciation led to lack of support in a multitude of settings. Additionally, CCS are often having to challenge hierarchal relationships to ask for support. For example, adolescent and young adult survivors of childhood cancers are at a point in their lives where they must fulfill role responsibilities as expected from parents, coaches, teachers, and bosses. They often described situations where they were not empowered or felt they were unequipped to challenge the role expectations held by leaders in their life. At home, when parents and other family members do not comprehend their limited ability to complete chores, the participant may be punished or suffer emotionally from the lack of support.

Well, I do know, like I was telling you earlier, my mom doesn't really understand it, and she complains all the time. ... It's hard. It's hard. It kind of makes me mad. (Participant 5)

At high school, one participant recalls difficulty participating in required physical activity. She felt that she had a true disability, but since she had the physical appearance that she was capable, she could not describe her limitations to her coaches. One participant describes the need to wear supportive footwear at her work setting, but her boss will ask her to change her shoes because they do not portray the image of the high-end retail store. Participants felt this lack of ability to explain their limitations and abilities resulted 


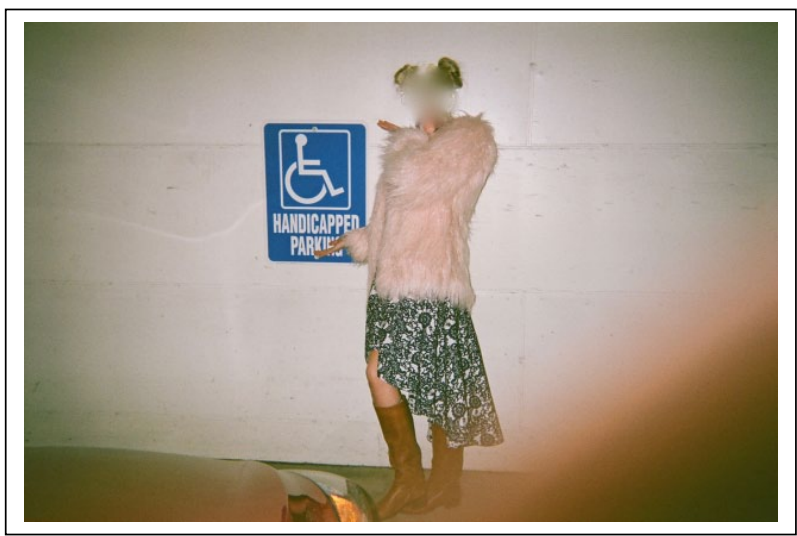

Figure 8. "I'm actually disabled, l'm not using my grandmother's tag."

in little modifications to their role responsibilities, and therefore, the survivors had to continue participating in tasks that they knew would elicit or worsen their symptoms. CIPN is described as an "invisible disability" that made it even more difficult for participants to ask for modifications to their expected responsibilities.

And because there's not a lot of words you can use to describe it to get your message across to people, so that they can fully comprehend what it's like for you (Participant 1)

In the community setting, participants expressed challenges they faced when they have been judged by people in the community. One participant was often verbally attacked for using handicap parking spaces, since people did not appreciate her disability visibly (see Figure 8). In social settings, one participant was kicked out of a bar while socializing with friends. She appeared to be intoxicated due to her abnormal gait caused by her severe bilateral foot drop. She was too embarrassed to explain that she was not intoxicated but rather that she had a disability. While experiencing judgment from those in the community was less distressing than experiencing lack of support from those who knew them well, it was a message that reinforced their disconnection from needed support.

CCS with CIPN also felt disconnected from medical support. CCS sensed that health care providers do not understand their condition, and as a result, CCS had low faith in interventions. These CCS had tried a variety of approaches for management, from medication to medical devices. Physical therapy was commonly reported, although few appreciated a direct benefit. One participant reported difficulty finding a physical therapist trained to care for CIPN. In addition, survivors felt that CIPN varied from other types of peripheral neuropathy, such as diabetic neuropathy, and reported a perceived lack of benefit from therapy that was generalized from other neuropathic conditions. As a result, participants often tried to cope on their own by using a variety of self-developed strategies. Some participants relied on exercise and stretching, while almost all had found unique strategies. For example, two participants reported using objects, such as a ball in their pocket or a wall, to touch to regain sensation or a sense of grounding.

And I feel like neuropathy is a very small box that you get put into.... And it's usually diabetic patients that I get put in a box with. . . . But I get put in this noncancerous, peripheral neuropathy box that has no bearing. (Participant 7)

\section{Discussion}

The purpose of this study was to illustrate the lived experience of CIPN among CCS. Findings show that CIPN is characterized as a condition of disconnection that results in broad functional and emotional impact. CIPN is a highly personal experience, where each participant had unique physical and emotional manifestations of disconnection. CCS may use a variety of symptom quality, timing, and intensity descriptors, while collectively conveying a sense of disconnection between mind and body. Symptom distress among participants is disproportionately low compared to the significant impact on physical performance and emotional outcomes. Many participants had resolved to "fight through it" and, as a result, present to their family, friends, community, and health care providers with little distress regarding their symptoms. This likely contributed to the disconnect survivors feel exists between their symptoms and the health care support they need. In a reciprocal way, participants sensed their health care providers underappreciate the significance of this condition. Many factors have likely contributed to the underappreciation of CIPN (Markman, 2006). These findings add to this literature by expanding what is known of the physical and emotional impact of CIPN among CCS, specifically in a population of adolescents and young adults.

To our knowledge, this is the first report of emotional implications of living with chronic CIPN among CCS. Disconnection experienced between mind and body causes frustration. Participants felt frustration "in the moment," as they attempted to achieve a certain task but failed as they could not control their bodily movements. Disconnection between expected potential and reality resulted in sadness and, in the worst outcomes, a feeling of worthlessness. Specifically, in this developmental period, adolescents and young adults can have significant negative outcomes if they are not able to fulfill their expected social roles (identity vs. role confusion) or make meaningful, connected relationships (identify vs. isolation; Erikson, 1993). The impact of disrupted normal developmental tasks distinguishes these findings from 
reports of adults with CIPN (Bakitas, 2007; Tofthagen, 2010a). Disconnection between survivors and support causes hopelessness. Oftentimes, participants report not discussing symptoms with their health care providers since they did not expect there were any effective treatment options. Due to the disconnection, CCS are often dealing alone with the emotional impact of CIPN.

The secondary purposes of this study were to demonstrate how CIPN affects school, vocational performance, and quality of life among CCS. All participants identified at least one impact on school and/or vocation, while many experienced a broad impact. In addition to challenges in physically participating in work-associated activities, CCS with CIPN are at risk for a poor career trajectory. This finding adds to previous work on vocational impact among adults with cancer who experience CIPN, by appreciating that the early age of treatment and the development of CIPN leads to earlier time of career impact. Earlier age at career impact would likely have a greater impact on their lifetime career trajectory. Adult cancer survivors with CIPN have previously reported difficulty with vocation and leisure activities (Bakitas, 2007; Tofthagen, 2010a). This study expands understanding by adding the perspective of developing adolescents and young adults.

\section{Strength and Limitations}

While this study adds to the literature by illuminating a poorly described health condition among CCS, the following limitations must be acknowledged. First, two of the seven participants were lost to follow-up. These two participants were called at the 2-week interval, and further questions regarding the photographic assignment were clarified. Each participant was called after the camera was not returned at the assigned 1-month interval. Both had changes in their current life situation that they identified as barriers to completing the photographic portion in a timely manner. Ultimately, the cameras were never returned, and interviews were not completed. The researcher reflected on what was ethically appropriate, and out of respect for these two participants, they were not asked to continue participation. While the researcher believes saturation was achieved, the potential for new findings among these two potential participants cannot be ruled out. Both participants lost to follow-up identified as African American, and the lack of their perspectives is an obvious weakness regarding the potential diversity of experiences that cannot be reported. Second, there were practical limitations to collecting photographic data. Unfortunately, two rolls of film were accidentally destroyed by the photo-processor. Fortunately, the participants who generated these photos either remembered significant images or took notes regarding their photographs, so we could discuss their photographs during the in-depth interview. Information regarding the destroyed photographs was volunteered by the participants, and they were not asked to recall this information. It must be acknowledged, however, that potentially photographs were taken that were not discussed during the interview. Also, the researcher-generated reflections on those images could not be made. Future researchers should consider utilizing digital photographic methods, where multiple copies can be created, stored, and shared with fewer risks to loss of data. Adolescents and young adult can be difficult to retain as research participants due to the transitions in their life and general lifestyle characteristics. To improve retention, future researchers should consider smart phones or digital cameras, which can be used with a secure digital drop box to allow for adolescents and young adults to participate from a distance and on their own schedule.

This research was strengthened by utilizing innovative methods in attempts to understand a phenomenon that is difficult to describe and measure. While previous studies have acknowledged the challenges of identifying and describing CIPN, photographs were unique tools to give voice to the experience that is otherwise not reported. This study also included patients who represent a variety of primary cancer diagnoses and thus a variety of chemotherapeutic exposures. Such variety broadens the understanding of this condition compared to previous studies that primarily utilize survivors of childhood leukemia (Jain et al., 2013; Lavoie Smith et al., 2015; Ramchandren et al., 2009). Lastly, the sample included patients many years from the completion of therapy, which provides a unique insight into the chronicity of this condition.

\section{Conclusion}

CCS experiencing CIPN long after the completion of cancer therapy are at risk for global functional and emotional impact on their daily lives. Symptom variability is present within and between patients, but the following overarching themes of disconnection summarizes the symptom experience: disconnect between mind and body, disconnect between anticipated potential and reality, and disconnect between survivors and support. Findings from this research support findings from previous studies of adult survivors with CIPN, that while symptoms improve for many patients after treatment has ended, a subset of patients have persistent and significantly impactful symptoms (Driessen et al., 2012; Tofthagen et al., 2012). This research expands on previous findings to illuminate the difference in symptom experience among those treated for cancer at a younger age and therefore dealing with chronic late effects of cancer therapy during crucial periods of development. Future researchers should utilize these findings to guide the development of interventions 
that support building the connections these survivors lack. Interventions should include emotional support, in addition to focusing on improved physical performance. Clinicians caring for CCS should evaluate their patients for CIPN, knowing that they are less likely to disclose their struggles with CIPN, and, at a minimum, offer strategies for emotional support to assist those living with this chronic condition as a result of their cancer therapy.

\section{Declaration of Conflicting Interests}

The author(s) declared no potential conflicts of interest with respect to the research, authorship, and/or publication of this article.

\section{Funding}

The author(s) disclosed receipt of the following financial support for the research, authorship, and/or publication of this article: Caroline Mohrmann was supported by the Doctoral Degree Scholarship in Cancer Nursing 127294-DSCN-15-080-01-SCN from the American Cancer Society. The Barnes-Jewish Hospital Foundation and Siteman Cancer Center also supported this research. Research reported in this publication was supported by the National Cancer Institute of the National Institutes of Health under Award Number R25CA190190. The content is solely the responsibility of the authors and does not necessarily represent the official views of the NIH.

\section{ORCID iD}

Caroline Mohrmann iD https://orcid.org/0000-0002-1198-0433

\section{References}

Armenian, S. H., Landier, W., Hudson, M. M., Robison, L. L., \& Bhatia, S. (2013). Children's Oncology Group's 2013 blueprint for research: Survivorship and outcomes. Pediatric Blood \& Cancer, 60, 1063-1068. doi:10.1002/ pbc. 24422

Armstrong, T., Almadrones, L., \& Gilbert, M. (2005). Chemotherapy-induced peripheral neuropathy. Oncology Nursing Forum, 32, 305-311. doi:10.1188/05.ONF.305-311

Bakitas, M. A. (2007). Background noise: the experience of chemotherapy-induced peripheral neuropathy. Nursing Research, 56, 323-331. doi:10.1097/01.NNR.0000289503.22414.79

Catalani, C., \& Minkler, M. (2010). Photovoice: A review of the literature in health and public health. Health Education \& Behavior, 37, 424-451. doi:10.1177/1090198109342084

Chung, T., Prasad, K., \& Lloyd, T. E. (2014). Peripheral neuropathy. Neuroimaging Clinics of North America, 24, 4965. doi:10.1016/j.nic.2013.03.023

Drew, S., \& Guillemin, M. (2014). From photographs to findings: Visual meaning-making and interpretive engagement in the analysis of participant-generated images. Visual Studies, 29, 54-67. doi:10.1080/1472586X.2014.862994

Driessen, C. M. L., de Kleine-Bolt, K. M. E., Vingerhoets, A., Mols, F., \& Vreugdenhil, G. (2012). Assessing the impact of chemotherapy-induced peripheral neurotoxicity on the quality of life of cancer patients. Supportive Care in Cancer, 20, 877-881.
Eckhoff, L., Knoop, A., Jensen, M. B., \& Ewertz, M. (2015). Persistence of docetaxel-induced neuropathy and impact on quality of life among breast cancer survivors. European Journal of Cancer (Oxford, England: 1990), 51, 292-300. doi:10.1016/j.ejca.2014.11.024

Emden, C. (1998). Theoretical perspectives on narrative inquiry. Collegian, 5(2), 30-35. doi:10.1016/S1322-7696 (08)60281-4

Erikson, E. H. (1993). Childhood and society. New York, NY: W. W. Norton.

Ezendam, N. P. M., Pijlman, B., Bhugwandass, C., Pruijt, J. F. M., Mols, F., Vos, M. C., . . .van de Poll-Franse, L. V. (2014). Chemotherapy-induced peripheral neuropathy and its impact on health-related quality of life among ovarian cancer survivors: Results from the population-based PROFILES registry. Gynecologic Oncology, 135, 510-517. doi:10.1016/j.ygyno.2014.09.016

Friedrichsdorf, S. J., \& Nugent, A. P. (2013). Management of neuropathic pain in children with cancer. Current Opinion in Supportive and Palliative Care, 7(2), 131-138. doi. org/10.1097/SPC.0b013e3283615ebe

Gilchrist, L. (2012). Chemotherapy-induced peripheral neuropathy in pediatric cancer patients. Seminars in Pediatric Neurology, 19, 9-17. doi:10.1016/j.spen.2012.02.011

Hagedorn, M. R. (1994). Hermeneutic photography: An innovative esthetic technique for generating data in nursing research. Advances in Nursing Science, 17, 44-50.

Hudson, M., Ness, K., Gurney, J., Mulrooney, D. A, Chemaitilly, W., Krull, K. R., . . Robison, L. L. (2013). Clinical ascertainment of health outcomes among adults treated for childhood cancer. JAMA, 309, 2371-2381. doi:10.1001/ jama.2013.6296

Jain, P., Gulati, S., Seth, R., Bakhshi, S., Toteja, G. S., \& Pandey, R. M. (2013). Vincristine-induced neuropathy in childhood ALL (acute lymphoblastic leukemia) survivors prevalence and electrophysiological characteristics. Journal of Child Neurology, 29, 932-937. doi:10.1177/0883073813491829

Kottschade, L. A., Sloan, J. A., Mazurczak, M. A., Johnson, D. B., Murphy, B. P., Rowland, K. M., . . . Loprinzi, C. L. (2010). The use of vitamin $\mathrm{E}$ for the prevention of chemotherapy-induced peripheral neuropathy: Results of a randomized phase III clinical trial. Supportive Care in Cancer, 19, 1769-1777. doi:10.1007/s00520-010-1018-3

Lavoie Smith, E. M., Li, L., Chiang, C., Thomas, K., Hutchinson, R. J., Wells, E. M., . . Renbarger, J. (2015). Patterns and severity of vincristine-induced peripheral neuropathy in children with acute lymphoblastic leukemia. Journal of the Peripheral Nervous System, 20, 37-46. doi:10.1111/jns. 12114

Lenz, E. R., Pugh, L. C., Milligan, R. A., Gift, A., \& Suppe, F. (1997). The middle-range theory of unpleasant symptoms: An update. Advances in Nursing Science, 19(3), 14-27.

Lopez, K. A., \& Willis, D. G. (2004). Descriptive versus interpretive phenomenology: Their contributions to nursing knowledge. Qualitative Health Research, 14, 726-735. doi:10.1177/1049732304263638

Markman, M. (2006). Chemotherapy-induced peripheral neuropathy: Underreported and underappreciated. Current Pain \& Headache Reports, 10, 275-278. 
Mohrmann, C., Armer, J., \& Hayashi, R. J. (2016). Challenges evaluating chemotherapy-induced peripheral neuropathy in childhood cancer survivors which instrument should nurses use? Journal of Pediatric Oncology Nursing, 34, 1-9.

Myers, J. S. (2009). A comparison of the theory of unpleasant symptoms and the conceptual model of chemotherapyrelated changes in cognitive function. Oncology Nursing Forum, 36, E1-E10. doi:10.1188/09.ONF.E1-E10

Ramchandren, S., Leonard, M., Mody, R. J., Donohue, J. E., Moyer, J., Hutchinson, R., \& Gurney, J. G. (2009). Peripheral neuropathy in survivors of childhood acute lymphoblastic leukemia. Journal of the Peripheral Nervous System, 14, 184-189. doi:10.1111/j.1529-8027.2009.00230.x

Richards, L., \& Morse, J. M. (2012). README FIRST for a user's guide to qualitative methods. Thousand Oaks, CA: Sage.

Sandelowski, M. (1991). Telling stories: Narrative approaches in qualitative research. Image: Journal of Nursing Scholarship, 23, 161-166.

Sandelowski, M. (1995). Sample size in qualitative research. Research in Nursing \& Health, 18, 179-183.

Seretny, M., Currie, G. L., Sena, E. S., Ramnarine, S., Grant, R., MacLeod, M. R., . . . Fallon, M. (2014). Incidence, prevalence, and predictors of chemotherapy-induced peripheral neuropathy: A systematic review and meta-analysis. PAIN, 155, 2461-2470. doi:10.1016/j.pain.2014.09.020

Tofthagen, C. (2010a). Patient perceptions associated with chemotherapy-induced peripheral neuropathy. Clinical Journal of Oncology Nursing, 14(3), E22-E28. doi:10.1188/10. CJON.E22-E28

Tofthagen, C. (2010b). Surviving chemotherapy for colon cancer and living with the consequences. Journal of Palliative Medicine, 13, 1389-1391. doi:10.1089/jpm.2010.0124

Tofthagen, C., Overcash, J., \& Kip, K. (2012). Falls in persons with chemotherapy-induced peripheral neuropathy. Supportive Care in Cancer, 20, 583-589.

Wampler, M. A., Topp, K. S., Miaskowski, C., Byl, N. N., Rugo, H. S., \& Hamel, K. (2007). Quantitative and clinical description of postural instability in women with breast cancer treated with taxane chemotherapy. Archives of Physical Medicine and Rehabilitation, 88, 1002-1008.

\section{Author Biography}

Caroline Mohrmann, $\mathrm{PhD}, \mathrm{RN}, \mathrm{CPNP}-\mathrm{AC}$, is a pediatric nurse practitioner in the Late Effects Clinic at Siteman Kids at Washington University. She is also a postdoctoral research associate in the Division of Public Health Sciences at Washington University. 